\title{
Why biodiversity matters
}

\author{
Welcome to the inaugural issue of Nature Ecology \& Evolution. Our mission is to bring you research and \\ comment that explore the diversity of life in all its grandeur and to promote the importance of ecology \\ and evolution in the wider world.
}

Scientists are not, generally speaking, activists. We hold strong views, care about the world and campaign for specific improvements, but we like to think we have objective neutrality that stops us crossing the line into activism. Two seemingly contradictory trends are blurring that distinction: we need to shout to be heard in a political discourse that is increasingly hostile to experts, and our ecological and evolutionary research is becoming more and more directly relevant to social and global concerns.

If we needed to sum up the interests of this journal and its readership in a single word, it would be biodiversity. From its origins in deep time, through its development, patterns and maintenance, to its preservation and importance to society, it is the thread that unites all of ecology and evolution. The policy relevance of the ecological side of biodiversity is obvious and well established. Human activity has caused a vast increase in the extinction rate, and the ecosystem services we depend on are severely threatened. We interview Anne Larigauderie (article no. 0036) from the Intergovernmental Science-Policy Platform on Biodiversity and Ecosystem Services (IPBES) and discuss how science and policy are being connected to address these threats. However, research on the evolutionary aspects of biodiversity is no less relevant, albeit less appreciated, and Wedell and Hosken argue (article no. 0035) that evolutionary biologists need to be better champions of the social importance of their work. For example, it is arguable that the antimicrobial resistance crisis, which is an evolutionary problem, rates alongside the climate and extinction crises in terms of existential threat to humanity. Beyond that, other medical topics such as cancer, obesity and infectious disease dynamics can only be properly understood in the light of evolution, and issues of global food security such as improving crop yields and managing sustainable fisheries are impossible to tackle without evolution as a central consideration.

The UN Sustainable Development Goals set out targets for many of these topics, and ecology and evolution have an important role in almost every goal. Fundamentally, the goals are about the ecological relationship between humanity and the planet, and how that relationship needs to change in order to achieve universal wellbeing. Goals 14 and 15, which cover life below water and life on land, respectively, are perhaps the most obviously tied to biodiversity, as Larigauderie mentions in her interview. However, we also take a look at some of the other goals in this issue: San Millan et al. (article no. 0010) and Ogbunugafor and Eppstein (article no. 0007) apply evolutionary biology to antimicrobial resistance (Goal 3), we have Bennett (article no. 0018) exploring the relationship between biodiversity, land use and food security (Goal 2), and Moran and Kanemoto (article no. 0023) investigate the effect of economic activity on biodiversity (Goals 8 and 12).

The twin pillars of this journal, ecology and evolution, have arguably always been inseparable, but the link between them has become tighter in recent years. As Pelletier describes (article no. 0034), in her review of Eco-Evolutionary Dynamics by Andrew Hendry, the interaction between the two disciplines is no longer considered a one-way street. Ecology doesn't just set up the landscape for evolution to occur in, but can be affected by evolutionary processes. The Articles by Faillace and Morin (article no. 0013) and Gloag et al. (article no. 0011), for example, demonstrate the importance of evolution in the context of ecological invasions, while Donovan et al. (article no. 0012) look at the interplay between evolutionary change and ecological interactions in the fossil record. The link between ecology and evolution is likely to get ever closer in the coming years with more data at even finer spatial and temporal scales.

This journal aims to be, first and foremost, a home for high calibre ecology and evolution research that is of wide interest across our research community. We also want to ensure that the disparate parts of that community interact with each other, across taxonomic, technical and time-scale boundaries. We enjoy the conversations between palaeoanthropologists and conservation biologists who are both interested in the relationship between humans and environment, or between biogeographers and molecular evolutionists who are interested in explaining diversity patterns in time and space. But why should any of this require us to be activists? In a world where the majority of policy was based on evidence and in which there was a universal level of high quality science education, we wouldn't need to be. The immediate applications of ecology and evolution research would be obvious to most, as would be the importance and inherent interest of more basic topics such as the evolution of organismal form (as explored by Martín-Durán et al., El Baidouri et al. and Clack et al.; article nos 0005, 0009 and 0002). But we don't live in that world. The recent US election has brought into positions of power individuals who doubt the truth of evolution and who might undermine decades of improvements in environmental protection. The environment has been a casualty in recent political decisions around the world such as in South America and Australia, and the Brexit vote in the UK highlighted a widespread distrust of academic experts.

One lesson from the turbulent political events of 2016 is that we need a deeper conversation between science, policy and the public. Our research should be embedded deep in the world-views of policymakers and public alike, not just as an optional add-on which can be rejected without significant consequences. The dramatic loss of biodiversity, the accelerating pace of climate change and the rise in untreatable infectious disease should be the backdrop to the majority of political conversations, not something confined to a science and environment silo. We scientists also need to be steeped in the mindsets of those who are not researchers, those who fund our work and whose lives it changes. We might not always need to take our activism to the streets, but we should be protesting bad policy decisions to those in political power and doing our utmost to engage with both public and policymakers alike about the central importance of ecology and evolution. 